\title{
Cosmic-ray slowing down in molecular clouds: Effects of heavy nuclei
}

\author{
Marin Chabot \\ Institut de Physique Nucléaire d'Orsay, IN2P3-CNRS and Université Paris-Sud, 91406 Orsay Cedex, France \\ e-mail: chabot@ipno.in2p3.fr
}

Received 1 December 2014 / Accepted 22 September 2015

\section{ABSTRACT}

\begin{abstract}
Context. A cosmic ray (CR) spectrum propagated through ISM contains very few low-energy $(<100 \mathrm{MeV})$ particles. Recently, a local $\mathrm{CR}$ spectrum, with strong low energy components, has been proposed to be responsible for the over production of $\mathrm{H}_{3}^{+}$molecule in some molecular clouds.

Aims. We aim to explore the effects of the chemical composition of low-energy cosmic rays (CRs) when they slow down in dense molecular clouds without magnetic fields. We considered both ionization and solid material processing rates.

Methods. We used galatic CR chemical composition from proton to iron. We propagated two types of CR spectra through a cloud made of $\mathrm{H}_{2}$ : those $\mathrm{CR}$ spectra with different contents of low energy CRs and those assumed to be initially identical for all CR species. The stopping and range of ions in matter (SRIM) package provided the necessary stopping powers. The ionization rates were computed with cross sections from recent semi-empirical laws, while effective cross sections were parametrized for solid processing rates using a power law of the stopping power (power 1 to 2 ).

Results. The relative contribution to the cloud ionization of proton and heavy CRs was found identical everywhere in the irradiated cloud, no matter which CR spectrum we used. As compared to classical calculations, using protons and high-energy behaviour of ionization processes $\left(\mathrm{Z}^{2}\right.$ scaling), we reduced absolute values of ionization rates by few a tens of percents but only in the case of spectrum with a high content of low-energy CRs. We found, using the same CR spectrum, the solid material processing rates to be reduced between the outer and inner part of thick cloud by a factor 10 (as in case of the ionization rates) or by a factor 100 , depending on the type of process
\end{abstract}

Key words. cosmic rays - ISM: clouds

\section{Introduction}

Cosmic rays (CRs) represent on average one fourth of the energy density in our galaxy interstellar medium (ISM; e.g. $1 \mathrm{ev} / \mathrm{cm}^{3}$, Lequeux et al. 2002). Those CRs with medium energy (i.e. $<10 \mathrm{GeV} /$ atomic mass unit $(\mathrm{GeV} / \mathrm{u}))$ are strongly coupled with other components of the ISM because they are ionizing particles within plasma structures undergoing magneto-hydrodynamic (MHD) behaviour at various scales. Because of their energies, magnetic confinement in the galaxy, (diffuse) ISM density, and processes of MHD reacceleration, it was acknoledged that on average the CRs travelling through ISM have a low-energy (i.e. $<100 \mathrm{MeV} / \mathrm{u}$ ) component of lower importance. This was confirmed for our local ISM with observations by the Voyager 1 spacecraft which is now out of the heliosphere (Stone et al. 2013). Recently, local incrementation of this low-energy part of the CR spectrum has been proposed to explain the observed variation at different locations in the ISM of the local abundance of $\mathrm{H}_{3}^{+}$signing $\mathrm{CR}$ ionization of $\mathrm{H}_{2}$ molecules (Indriolo et al. 2007, 2009; Padovani et al. 2009). Nuclear gamma ray measurements with INTEGRAL (Tatischeff et al. 2012) also indicated that local modulation of the low-energy CR is a possibility. At low energy, ionization cross sections no longer scale with the square of the CR nucleus charge like at high energy. The usual incrementation of the ionization rates due protons with a constant factor taking into account the heavy CR components (Spitzer \& Tomasko 1968) is then uncertain.
We aim to discuss the effect of the CR chemical composition when CRs with a strong low-energy component interact with a dense molecular cloud. We present the physics and calculations needed to propagate CR through $\mathrm{H}_{2}$ in Sects. 2 and 3. We present ionization rate calculations and discuss their evolution within a dense cloud in Sect. 4. We present and discuss dust processing rates by CR in Sect. 5 and, finally, we provide conclusions.

\section{Slowing down of charged particles}

A particle of nuclear charge $Z$, mass $A$ and energy $E$, passing through a slice of matter loses energy. The quantity that qualifies this energy loss is the stopping power $\mathrm{d} E / \mathrm{d} x$, which gives the energy loss by unit of target matter thickness expressed in atoms or molecules/area. Disregarding both high energies, where nuclear reactions play a major role, and very low energies, where elastic collisions have to be considered, the slowing down is dominated by the electronic excitations/ionizations of the target. Figure 1 shows the $\mathrm{d} E / \mathrm{d} x$ in $\mathrm{H}_{2}$ gas as a function of the energy per atomic mass unit (u) of all considered CR species (proton to iron; The stopping and range of ions in matter (SRIM) package, Ziegler et al. 2010). We assume the $\mathrm{H}_{2}$ target to form the bulk of ISM clouds, that is, the $10 \% \mathrm{He}$ atom mixed within is ignored, but this He atom may be taken into account by replacing the target thickness by an augmented (1.1) effective target thickness. The maximum of the $\mathrm{d} E / \mathrm{d} x$ does not occur at the same energy per atomic 


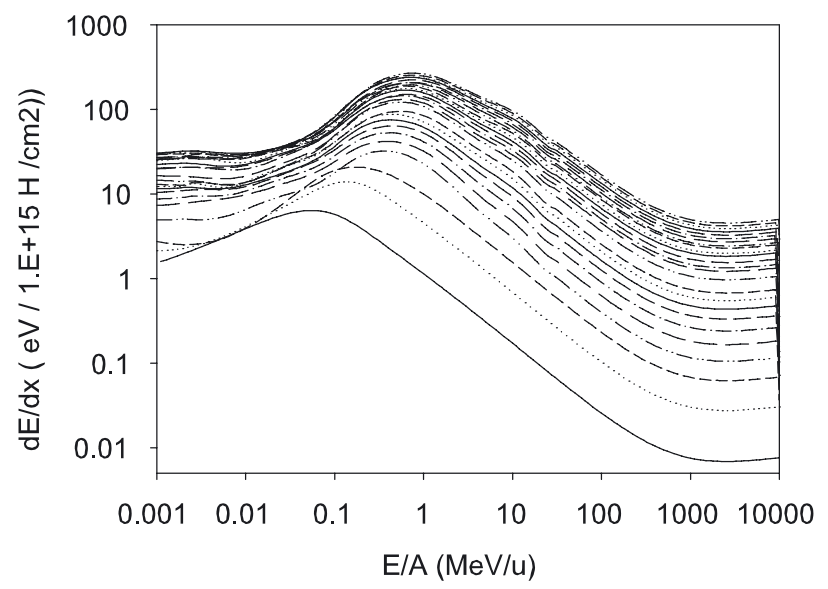

Fig. 1. Stopping power $\mathrm{d} E / \mathrm{d} x$ of the CR components from proton (lower curve) to iron (upper curve) in gaseous $\mathrm{H}_{2}$ target taken from SRIM package (Ziegler et al. 2010).

mass unit for the different species. Indeed, $\mathrm{d} E / \mathrm{d} x$ scales as:

$E / A_{\max } \sim Z+1$,

while maximum of the $\mathrm{d} E / \mathrm{d} x$ is scaling as

$\mathrm{d} E / \mathrm{d} x_{\max } \sim Z$.

\section{Propagation}

The quantity to be computed is the local CR spectrum $J_{Z}(E / A, X)$. This quantity gives the number of particles $(Z, A)$ of energy $E$ per unit time, area, energy per atomic mass unit, and solid angle at a particular place $X$ from the surface (expressed in matter thickness's unit $\left.\left(\mathrm{H}_{2} / \mathrm{cm}^{2}\right)\right)$ of the cloud. The location $X$ is only the column density $N\left(\mathrm{H}_{2}\right)$. The CR spectrum $J_{Z}(E / A, X)$ depends on the incident CR spectrum at the surface of the cloud $J_{Z}(E / A, 0)$, according to Padovani et al. (2009), Cecchi-Pestellini \& Aiello (1992),

$J_{Z}(E / A, X)=J_{Z}\left(E_{0} / A, 0\right) \frac{\mathrm{d} E / \mathrm{d} x\left(E_{0}\right)}{\mathrm{d} E / \mathrm{d} x(E)}$,

where $E_{0}$ is the incident energy at the cloud entrance that corresponds to the energy $E$ at the cloud depth $X$.

The incident CR spectra at the cloud entrance was assumed to be identical for all CR species,

$J_{Z}\left(E_{0} / A, 0\right)=\frac{f_{Z}}{f_{Z=1}} J_{1}\left(E_{0} / A, 0\right)$,

where $f_{Z}$ are the fractional abundances of the species $(Z, A)$ and $J_{1}$ is the initial incident CR spectrum at the entrance of the cloud for proton.

Elementary fractional abundances of CR were taken from George et al. (2009) and correspond to local Galactic CR (GCR) abundances measured on Earth above the energy of exclusion of the low-energy CRs by the solar heliosphere. In comparision with the solar chemical composition of $\mathrm{CR}$, the GCR contains many more heavy CRs.

\subsection{Incident CR spectra}

The full lines in Figs. 2 and 3 indicate the two different incident CR spectra $J_{1}\left(E_{0} / A, 0\right)$ used in the forthcoming calculations. The first initial $J_{1}\left(E_{0} / A, 0\right)$ CR spectrum (Fig. 2) is from
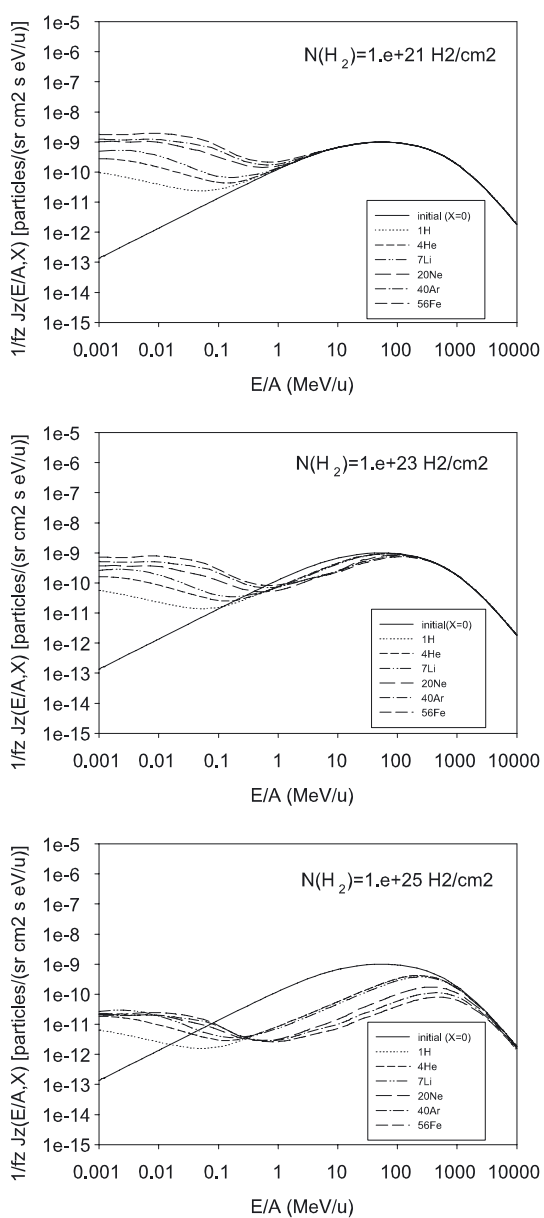

Fig. 2. Spectral density of some CRs $\left(\mathrm{p},{ }^{4} \mathrm{He},{ }^{7} \mathrm{Li},{ }^{12} \mathrm{C},{ }^{20} \mathrm{Ne},{ }^{40} \mathrm{Ar},{ }^{56} \mathrm{Fe}\right)$ at different cloud depths (upper pannel: $10^{+21} \mathrm{H}_{2} / \mathrm{cm}^{2}$, middle panel: $10^{+23} \mathrm{H}_{2} / \mathrm{cm}^{2}$ ) and for a thick target (lower panel: $10^{+25} \mathrm{H}_{2} / \mathrm{cm}^{2}$ ). The full line in all panels gives the SP68 initial CR spectrum at the surface of the cloud taken from Spitzer \& Tomasko (1968).

Spitzer \& Tomasko (1968). This spectrum is found to be in rough agreement with the output of CR reacceleration models, such as GALPROP (Vladimirov et al. 2011), and with our local Galactic CR spectrum (Stone et al. 2013). This spectrum introduces a low content of low-energy CRs; we refer to it as SP68. The second initial CR spectrum (Fig. 3) has a large, low-energy CR content compared to the first spectrum. This second spectrum is one of the typical CR spectra recently proposed (Padovani et al. 2009; Indriolo et al. 2009) to explain the strong $\mathrm{H}_{3}^{+}$molecule abundances observed in some diffuse clouds (Indriolo et al. 2007). The second spectrum has an $E / A^{-1}$ function for $E / A$ lower than $300 \mathrm{MeV} / \mathrm{u}$, joining the $\mathrm{CR}$ spectrum of Webber \& Yushak (1983) (with the form parameter $T_{0}=300 \mathrm{MeV} / \mathrm{u}$ ) for $\mathrm{E} / \mathrm{A}$ higher than $300 \mathrm{MeV} / \mathrm{u}$. We refer to it as the " $E^{-1}$ " $\mathrm{CR}$ spectrum.

\subsection{Evolution of $C R$ spectra within the cloud}

Figures 2 and 3 show the $\frac{f_{Z=1}}{f_{Z}} J_{Z}(E / A, X)$ CR spectra at three different places in the cloud. We obtained these spectra numerically using Eq. (3). For readability, we only show a few CR species. Each CR spectrum seems to evolve at large depths towards a unique curve independent of the initial spectrum. The shape of this "asymptotic" curve is related to the $\mathrm{d} E / \mathrm{d} x$ shape (Padovani et al. 2009) peculiar to each $(Z, A)$ values. 
M. Chabot: Cosmic-ray slowing down in molecular clouds: Effects of heavy nuclei
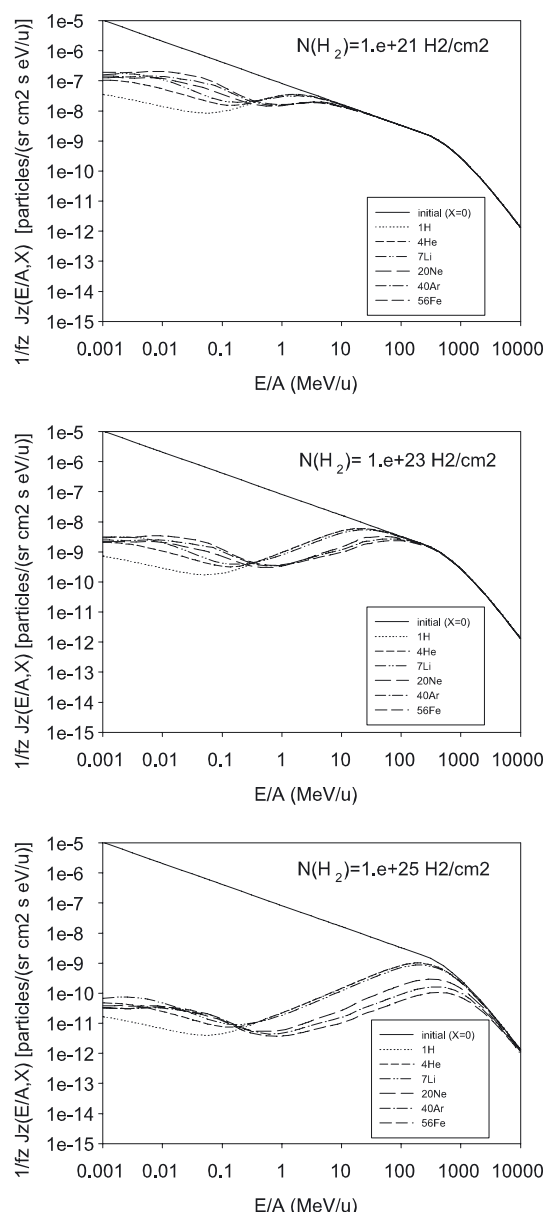

Fig. 3. Spectral density of some CRs $\left(\mathrm{p},{ }^{4} \mathrm{He},{ }^{7} \mathrm{Li},{ }^{12} \mathrm{C},{ }^{20} \mathrm{Ne},{ }^{40} \mathrm{Ar},{ }^{56} \mathrm{Fe}\right)$ at different cloud depths (upper pannel: $10^{+21} \mathrm{H}_{2} / \mathrm{cm}^{2}$, middle panel: $10^{+23} \mathrm{H}_{2} / \mathrm{cm}^{2}$ ) and for a thick target (lower panel: $10^{+25} \mathrm{H}_{2} / \mathrm{cm}^{2}$ ). The full line in all panels gives the $E^{-1}$ initial CR spectrum at the surface of the cloud(see text).

Figure 4 gives the evolution of the total CR chemical composition relatively to the initial CR chemical composition $\chi_{R}(X)$ defined as

$\chi_{R}(X)=\frac{f_{z=1}}{f_{z}} \frac{\int_{1 \mathrm{keV} / \mathrm{u}}^{10 \mathrm{GeV} / \mathrm{u}} J_{Z}(E / A, X) \mathrm{d}(E / A)}{\int_{1 \mathrm{keV} / \mathrm{u}}^{10 \mathrm{GeV} / \mathrm{u}} J_{1}(E / A, X) \mathrm{d}(E / A)}$.

Using the SP68 CR spectrum, the effect of the slowing down on the CR composition is negligible even at column density $\approx 10^{23} \mathrm{H}_{2} / \mathrm{cm}^{2}$, which corresponds to thick clouds. This is because the shape of SP68 is very close (with exception of the small contribution of the very low-energy part) to the CR shape obtained for a moderately thick target (see Fig. 2, top and middle). For the $E^{-1}$ spectrum, the effect of the slowing down on the total CR composition is small (reduction of heaviest species by a factor 2 at column density $\approx 10^{23} \mathrm{H}_{2} / \mathrm{cm}^{2}$ ). For very thick targets (e.g. $\approx 10^{25} \mathrm{H}_{2} / \mathrm{cm}^{2}$ ), the CR composition it is affected by the slowing down (reduction of heaviest species by one order of magnitude at $\approx 10^{25} \mathrm{H}_{2} / \mathrm{cm}^{2}$ ). These kinds of very thick targets may only be found in massive protostellar collapse of clouds. In these cases, if a source of CR with a strong low-energy component is embedded within the cloud, the chemical composition of the CR reinjected in the ISM may differ significantly from the $\mathrm{CR}$ composition at the $\mathrm{CR}$ source.
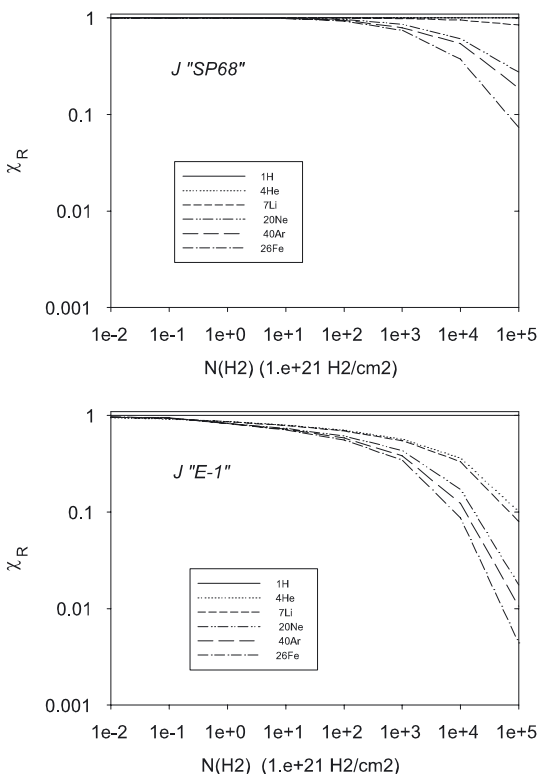

Fig. 4. Evolution of the $\mathrm{CR}$ species abundances, normalized to their values at the entrance of the cloud as function of the cloud depth (Eq. (5)). Upper panel: incident SP68 CR spectrum; lower panel: incident $E^{-1}$ spectrum.

\section{Ionization rate}

The ionization rate of $\mathrm{H}_{2}$ molecule by $\mathrm{CR}(Z, A)$ is written

$\zeta(X)=$

$4 \pi \sum_{Z} \int_{E / A_{\min }}^{E / A_{\max }} J_{Z}(E / A, X) \sigma_{\mathrm{ion}}(Z, E / A)\left(1+\phi_{\mathrm{s}}(Z, E / A)\right) \mathrm{d}(E / A)$,

where $\sigma_{\text {ion }}(Z, E / A)$ is the ionization cross section of $\mathrm{H}_{2}$ by a projectile $(Z, A)$ with an energy $E$, and $\phi_{\mathrm{s}}(Z, E / A)$ is a coefficent representing the contribution of the secondary electrons to the total ionization cross sections. The upper integration limit $E / A_{\max }$ is taken to be $10 \mathrm{GeV} / \mathrm{u}$ and the lower limit $E / A_{\min }$ as 1 $\mathrm{keV} / \mathrm{u}$. Indeed, outside these limits, the integral is small due to either cross sections and/or spectral density.

The classical calculation of the ionization rate (Spitzer \& Tomasko 1968; Padovani et al. 2009) assumes that i) the secondary electron contribution to total ionization is identical for all CR species; and ii) all quantities involving the interaction of a $\mathrm{Z}$ projectiles with $\mathrm{H}_{2}$ (e.g. stopping power and ionization cross sections) scale as $Z^{2}$.

Introducing the relative contribution to ionization of proton and heavy CRs,

$\eta=\frac{\zeta(X)_{Z>1}}{\zeta(X)_{Z=1}}$

It is easy to show that the classical ionization rate $\zeta(X)_{\mathrm{C}}$ is expressed as

$\zeta(X)_{\mathrm{C}}=\left(1+\eta_{\mathrm{C}}\right) \zeta(X)_{Z=1}$

with $\eta_{\mathrm{C}}$, which is independent of $X$, and is written as

$\eta_{\mathrm{C}}=\sum_{Z=2}^{Z=26} \frac{f_{z}}{f_{z=1}} Z^{2}$

With the abundances of George et al. (2009), the classical contribution of heavy $\mathrm{CR} \eta_{\mathrm{C}}$ is found to be 1.9 . 


\subsection{Secondary electron coefficent $\phi_{\mathrm{S}}(Z, E)$}

The goal of this section is to check whether the secondary electron contribution to the total ionization is identical for all CR species or has to be treated independently for each CR species.

The secondary electron coefficent is written as follows:

$\phi_{\mathrm{s}}(Z, E)=\frac{\int_{\mathrm{IP}\left(\mathrm{H}_{2}\right)}^{4 E / A} \operatorname{SDCS}_{Z}\left(E, E_{\mathrm{s}}\right) P_{\text {ion }}\left(E_{\mathrm{s}}\right) \mathrm{d} E_{\mathrm{s}}}{\int_{0}^{4 E / A} \operatorname{SDCS}_{Z}\left(E, E_{\mathrm{s}}\right) \mathrm{d} E_{\mathrm{s}}}$.

where $\operatorname{SDCS}_{Z}\left(E, E_{\mathrm{S}}\right)$ is the single differential cross section with respect to the energy of the ejected electrons $E_{\mathrm{S}}$ for a CR projectile $(Z, A)$ with an energy $E$ and $P_{\text {ion }}\left(E_{\mathrm{s}}\right)$ is the total probality of ionizing $\mathrm{H}_{2}$ with an electron of energy $E_{\mathrm{S}}$,

$P_{\text {ion }}\left(E_{\mathrm{s}}\right)=\int_{0}^{R\left(E_{\mathrm{s}}\right)} \sigma_{\text {ion }}(E(X)) \mathrm{d} X$

with $R\left(E_{\mathrm{s}}\right)$ the range in $\mathrm{H}_{2}$ target of an electron with an energy $E_{\mathrm{s}}$, and $E(X)$ the energy of this electron when passing through a $\mathrm{H}_{2}$ target of thickness $X$. The upper limit in the integration of (10) is given by the two-body kinematics (CR vs. electron) and the lower limit takes the ionization potential (IP) of the target electrons into account.

For CR protons, pioneer calculations (Glassgold \& Langer 1973; Cravens \& Dalgarno 1978) used SDCS deduced from experimental measurements (Opal et al. 1972, 1971) using electron projectiles instead of protons (e.g. scaled in velocity). Calculations made with this SDCS are referred as to $\mathrm{SDCS}_{\mathrm{e}}$. A semi-empirical law based on many SDCS measurements with proton projectiles has been established by Rudd et al. (1992). Calculations made with this SDCS are referred to as $\mathrm{SDCS}_{\mathrm{p}}$. For bare $Z$ projectiles, we found only one semi-empirical law adjusted for only a few experimental SDCSs (Stolterfoht et al. 1997). Calculations performed with this SDCS are referred to as $\mathrm{SDCS}_{z}$. For partially stripped projectiles that are relevant for low-energy CR (see below), we did not find any quantitative SDCS studies. Qualitatively, two effects may distort the SDCS of bare ions: the saturation effect, which is a deviation from the $Z^{2}$ law occuring at large pertubation $K \sim \frac{\text { charge }}{\text { velocity }}$ intensity, (Chabot et al. 1994; Wohrer et al. 1986) and the well-known screening effect (McGuire et al. 1981), which takes into account the screening on the nuclear charge by the bound projectile electrons in the excitation/ionization of the target. For the stopping power of heavy projectiles at equilibrium charge state in $\mathrm{H}_{2}$, these two corrections were found acting in an opposite way (Chabot et al. 2007). The SDCS for bare or partially stripped ions are likely to behave similarly.

The stopping power of electrons in $\mathrm{H}_{2}$, needed to compute $R\left(E_{\mathrm{s}}\right)$ in formula (11), was taken from measurements of Muñoz et al. (2007). Ionization cross sections of $\mathrm{H}_{2}$ by electrons, used in the same Eq. (11), were taken from measurements of Straub et al. (1996). We used an $E^{-1}$ law to extend these two quantities to electron energies higher than a few $\mathrm{keV}$, which is of lesser importance for the total ionization.

The secondary electron ionization $\phi_{\mathrm{s}}^{\mathrm{SDCS}}$ coefficients for the three SDCS (e.g. scaled electron, experimental proton, experimental bare projectiles) are listed in Table 1 for various incident CR energies.

Table 1 also lists the secondary electron mean energies $\left(\bar{E}_{\mathrm{s}}\right)$ defined as

$\bar{E}_{\mathrm{s}}(E)=\frac{\int_{0}^{4 E / A} \operatorname{SDCS}\left(E, E_{\mathrm{s}}\right) E_{\mathrm{s}} \mathrm{d} E_{\mathrm{s}}}{\int_{0}^{4 E / A} \operatorname{SDCS}\left(E, E_{\mathrm{s}}\right) \mathrm{d} E_{\mathrm{s}}}$.

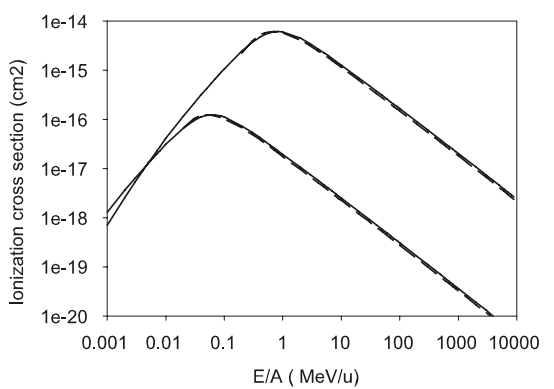

Fig. 5. Ionization cross sections of $\mathrm{H}_{2}$ by proton and ${ }^{56} \mathrm{Fe}$ projectiles as function of the energy per $u$. The upper curves correspond to Fe projectiles and the lower curves to proton. Full lines correspond to Rudd et al. (1983) semi-empirical law (formula (17)) scaled as explained in the text and dashed lines to the semi-empirical law (formula (82)) of Kaganovich et al. (2006).

The $\mathrm{SDCS}_{\mathrm{e}}$ and $\mathrm{SDCS}_{Z}$ show a moderate dependency for both $\bar{E}_{\mathrm{S}}$ and $\phi_{\mathrm{s}}$ with the CR energy, while $\mathrm{SDCS}_{\mathrm{p}}$ predicts an almost constant value for energies larger than about $1 \mathrm{MeV} / \mathrm{u}$. All SDCSs give a $\phi_{\mathrm{s}}$ value of $0.7 \pm 0.2$ in the energy range 1 $\mathrm{MeV} / \mathrm{u}-100 \mathrm{MeV} / \mathrm{u}$, which is the part of the CR spectrum that contributes most to the ionization.

It follows that the canonical proton value of $\phi_{\mathrm{s}} \approx 0.7$ (Spitzer \& Tomasko 1968) is also found to apply for heavy CR at all energies. We then rewrite the ionization rate given by the Eq. (6)

$\zeta(X)=\left(1+\phi_{\mathrm{s}}\right) 4 \pi \sum_{Z} \int J_{Z}(E / A, X) \sigma_{\mathrm{ion}}(Z, E / A) \mathrm{d}(E / A)$.

\subsection{Ionization cross sections}

The full lines in Fig. 5 give the cross sections used for computing the ionization rates for the two extreme CR species (proton and iron).

The cross sections for protons are from Rudd et al. (1983) using a semi-empirical law (Eq. (17)) based on exhaustive measurements for the $\mathrm{p}+\mathrm{H}_{2}$ collision. For $Z$ projectiles, Kaganovich et al. (2006) give a semi-empirical fit (Eq. (82)) adjusted by a compilation of measurements. The dashed line in Fig. 5 shows this semi-empirical law. Since this semi-empirical does not cover all the low energies involved in the calculations, we used the semi-empirical law of Rudd et al. (1983) scaled with the following two parameters:

$\left.\sigma_{\text {ion }}(Z, E / A)=\sigma_{\text {ion }}^{\text {Rudd83 }}(E / A) \times \frac{E_{Z=1}^{\max }}{E_{Z}^{\max }}\right) \times \frac{\sigma_{\text {ion }}^{\max }(Z, E / A)}{\sigma_{\text {ion }}^{\max }(Z=1, E / A)}$,

where $\sigma_{\text {ion }}^{\max }(Z, E / A)$ is the maximum value of the cross section (Kaganovich et al. 2006 Eq. (80)), which occurs at the energy $E_{Z}^{\max }$ (Kaganovich et al. 2006 Eq. (81)). The full line in Fig. 5 shows the result of this scaled law.

We do not take into account the ionization of $\mathrm{H}_{2}$ by the capture process at very low energy $\left(\mathrm{H}^{+}+\mathrm{H}_{2}->\mathrm{H}+\mathrm{H}_{2}^{+}\right)$as Padovani et al. (2009) because, as seen in Fig. 6, the average charge state of the slowing down proton is almost zero at low energy and the electron capture on $\mathrm{H}_{2},\left(\mathrm{H}+\mathrm{H}_{2}->\mathrm{H}^{-}+\mathrm{H}_{2}^{+}\right)$reduced by more than a factor 10 (Williams 1967). Nevertheless, this process is of little importance with respect to the spectral densities at those very low energies inside clouds. 
Table 1. Mean energy $(\mathrm{eV})$ of secondary electrons $\left(\bar{E}_{\mathrm{s}}\right)$ and secondary electron coefficient $\phi_{\mathrm{s}}$ for different projectile impact velocity, using three different types of single differential cross section (SDCS) (see text).

\begin{tabular}{ccccccc}
\hline \hline$E / A(\mathrm{MeV} / \mathrm{u})$ & $\bar{E}_{\mathrm{s}}^{\mathrm{SDCS}_{\mathrm{e}}}$ & $\bar{E}_{\mathrm{s}}^{\mathrm{SDCS}_{\mathrm{p}}}$ & $\bar{E}_{\mathrm{s}}^{\mathrm{SDCS}_{\mathrm{Z}}}$ & $\phi_{\mathrm{s}}^{\mathrm{SDCS}_{\mathrm{e}}}$ & $\phi_{\mathrm{s}}^{\mathrm{SDCS}_{\mathrm{p}}}$ & $\phi_{\mathrm{s}}^{\mathrm{SDCS}_{\mathrm{Z}}}$ \\
\hline 0.1 & 15.3 & 27.6 & 23.5 & 0.39 & 0.34 & 0.44 \\
1.0 & 25.1 & 37.3 & 34.0 & 0.42 & 0.69 & 0.64 \\
10. & 35.1 & 39.8 & 41.6 & 0.63 & 0.73 & 0.79 \\
100. & 45.1 & 41.5 & 47.6 & 0.80 & 0.72 & 0.87 \\
1000. & 55.7 & 42.7 & 52.8 & 0.88 & 0.69 & 0.91 \\
\hline
\end{tabular}

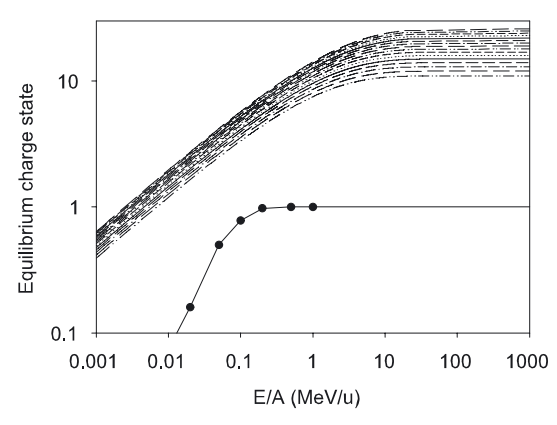

Fig. 6. Equilibrium charge state for proton from measurements of Stier \& Barnett (1956) (symbols) and for heavy CRs (sodium to iron) from Betz (1972) (Eq. (5.7)) (lines). Betz's equation underestimated results for $Z<35$.

\subsection{Evolution of ionization rate within the cloud}

Figure 7 presents the evolution of the ionization rates as a function of the penetration in the cloud. The two initial CR spectra SP68 and $E^{-1}$ were used in the full calculation (Eq. (13)) and in the classical calculation (Eq. (8)). We performed integrations from $1 \mathrm{keV}$ to $10 \mathrm{GeV}$.

When compared to SP68, the $E^{-1} \mathrm{CR}$ spectrum has a large proportion of low-energy $\mathrm{CR}$ (with strong ionizing cross sections) and produces many more ionizations at the entrance of the cloud. As the target thickness increases, these low-energy components are efficiently suppressed. For extremely large thicknesses, the ionization rate becomes independent of initial spectrum because only the high-energy CRs, identical between the $E^{-1}$ and SP68 spectrum, have survived (Padovani et al. 2009).

For both initial CR spectra, the classical calculation (C) is always above the full calculation $(\mathrm{F})$. This is understandable since ionization cross sections induced by heavy CR are smaller than cross sections obtained by scaling with $Z^{2}$ the proton ionization cross sections (see Fig. 5). The effect of the full calculation is small (reduction by few tens of percents for the $E^{-1}$ spectrum) and almost independent of the position inside the cloud. Since the classical calculation overestimates ionization by heavy nuclei $\left(0<\eta<\eta_{\mathrm{C}}=2\right)$, reduction of ionization rate by the full calculation cannot exceed $3\left(\frac{\zeta_{\mathrm{F}}}{\zeta_{\mathrm{C}}}=(1+\eta) /\left(1+\eta_{\mathrm{C}}\right)<1 / 3\right)$.

\section{Solid processing rates}

The CRs entering molecular clouds interact with dust grains. Grain microstructure may then be modified and some materials (atoms and/or molecules) may be released in the gas phase. An important parameter governing these processes is the size of the grains. Indeed, if the grain is small enough, the deposited energy by a single CR leads to a temperature rise of the whole grain. In contrast, if the grain is large enough, the temperature stays constant and we only consider processes in the track of

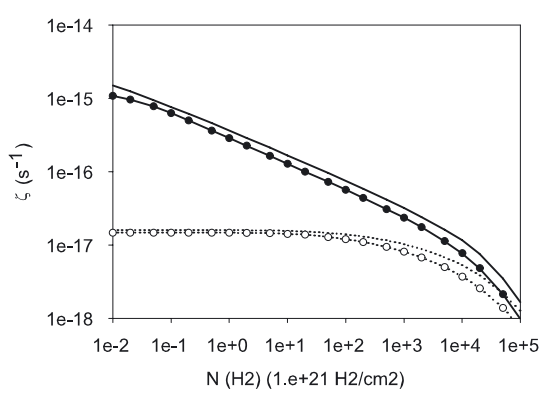

Fig. 7. Ionization rates as a function of the column density. Full lines correspond to calculations using the $E^{-1} \mathrm{CR}$ spectrum, and dotted lines to calculations using the SP68 CR spectrum. Lines with symbols correspond to the full calculations and lines without symbols to the classical calculation assuming $\mathrm{Z}^{2}$ scaling of all processes.

the ion passing (or stopping ) through (in) the solid. The diameter of this track is approximately one to a few micrometers, depending of the kind of the process under interest. For very small grains containing less that a few hundred of atoms multiionization processes have been recognized as very sensitive to heavy CR (M. Chabot, Colloque PCMI 2004 using Wohrer et al. 1994, unpublished).

\subsection{Structural modification and, desorption from large grains}

We define an effective cross section in order to describe the effects of CRs for a given material and for a given process. This cross section is written as a power-law function of the stopping power. Indeed, for a given solid density and considering a unique track diameter related to the process under interest, the stopping power gives the energy per "atomic" constituent of the material, the relevant quantity for microscopic modifications (e.g. Godard et al. 2011). Effective cross sections are accordingly written as follows:

$\sigma_{\mathrm{eff}}(Z, E / A) \propto \mathrm{d} E / \mathrm{d} x(Z, E / A)^{\alpha}$.

As we defined effective cross sections, we calculated effective rates by replacing in formula $(13) \sigma_{\text {ion }}(Z, E / A)$ by $\sigma_{\text {eff }}(Z, E / A)$ and setting the secondary electron coefficient $\phi$. to zero. We report on calculations performed with $\mathrm{d} E / \mathrm{d} x$ powers $\alpha=1$ and $\alpha=2$. For bulk modifications, such as dehydrogenation of carbonaceous material (Godard et al. 2011), or ice compaction (Dartois et al. 2013), the slope $\alpha$ has been found close to unity in experiments. For desorption processes (i.e. release of material in the gas phase) a slope $\alpha$ of two has been reported (Molvik et al. 2007; Dartois et al. 2015).

Figure 8 gives the material modification rates as a function of the column density for the two different values of $\alpha$ for the two initial CR spectrum, $E^{-1}$ and SP68. The rates have been normalized to those obtained with the SP68 CR spectrum at the entrance of the cloud with $\alpha=1$. 


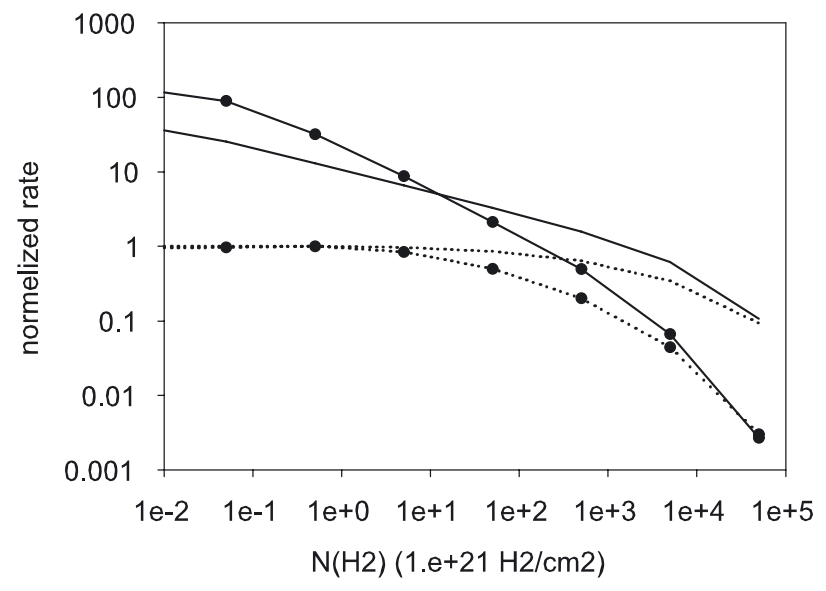

Fig. 8. Material processing normalized rates as a function of the column density. Full lines correspond to calculations using the $E^{-1} \mathrm{CR}$ spectrum, and dotted lines to calculations using the SP68 CR spectrum. Lines with symbols correspond to desorption $(\alpha=2)$ processes and lines without symbols to bulk modifications $(\alpha=1)$.

For $\alpha=1$, the same behaviour as observed for the ionzation rates is occuring because ionization cross sections are strictly proportional to the stopping power ( e.g. $\sigma_{\text {ion }}(Z, E / A) \propto$ $\mathrm{d} E / \mathrm{d} x(Z, E / A))$. This is because ionization and electronic excitations are responsible for the stopping power and are almost in a constant proportion regardless of the velocity. The exception arises in the very low velocity regime where ionization is becoming weaker when compared to excitation, but of lower importance as already discussed. The rates are uniform across the cloud for the SP68 spectrum, while for the $E^{-1}$ spectrum rates at the outer and the inner part $\left.\left(\approx 10^{23} \mathrm{H}_{2} / \mathrm{cm}^{2}\right)\right)$ of the cloud differs by more than one order of magnitude.

For $\alpha=2$, the importance of the heavy CR slowing down is strongly reinforced. Using the SP68 CR spectrum, there is a factor two between the entrance $\left(10^{+19} \mathrm{H}_{2} / \mathrm{cm}^{2}\right)$ and the depth of a thick cloud $\left(10^{+23} \mathrm{H}_{2} / \mathrm{cm}^{2}\right)$, and there are almost two orders of magnitude differences for the $E^{-1}$ spectrum.

\subsection{CR processes on finite size grains}

The sub-micrometer grains on the inside of the cloud covered by volatile molecules that condense on them may experience a whole temperature rise due to a single CR impact (Leger et al. 1985; D'Hendecourt et al. 1982). Release of (condensed) material in the gas phase may then occur. The rate of release (molecules grain ${ }^{-1} \mathrm{~s}^{-1}$ ) for a grain of radius $a$ is given by Shen et al. (2004),

$R(X)=4 \pi \sum_{Z} \int J_{Z}(E / A, X) \pi a^{2} N(\Delta E) \mathrm{d}(E / A)$.

Where $N(\Delta E)$ is the number of molecules released in the gaz phase for an energy deposit by the CR inside a grain $\Delta E$.

To derive numerical (relative) rates with SP68 and $E^{-1}$ CR spectra, we used the model of grain heating of Leger et al. (1985) in absence of UV with the values computed for $0.1 \mu \mathrm{m}$ grain by Shen et al. (2004). We state that

$N(\Delta E) \sim \Delta E-22.7 \mathrm{keV}$,

with

$\Delta E=\int_{0}^{a \rho} \mathrm{d} E / \mathrm{d} x_{\mathrm{si}}(x) \mathrm{d} x$,

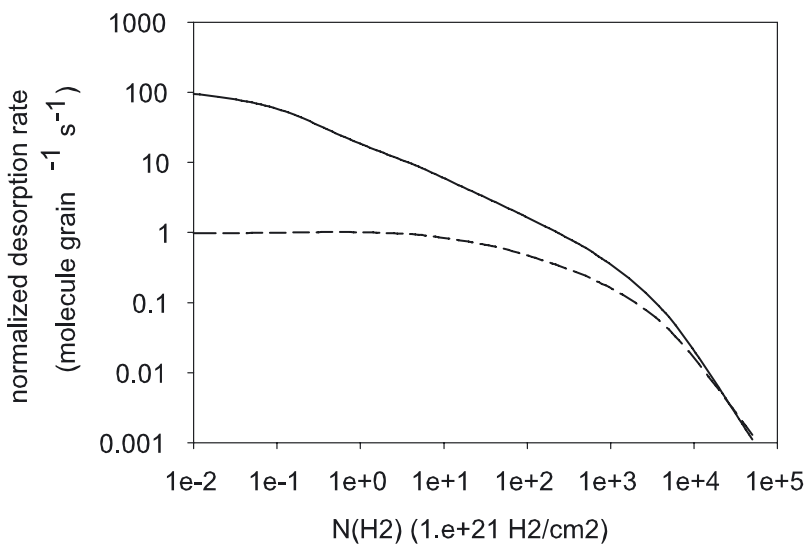

Fig. 9. Thermal desorption rates induced by $\mathrm{CR}$ as a function of the column density using SP68 CR spectrum (dashed line)and $E^{-1}$ CR spectrum (full line). The rates are normalized to the rate obtained with the SP68 CR spectrum at low column density.

where a is the grain radius $(0.1 \mu \mathrm{m}), \rho$ is the density of silicum $\left(5 \times 10^{22}\right.$ atoms $\left./ \mathrm{cm}^{3}\right)$, and $\mathrm{d} E / \mathrm{d} x_{\mathrm{si}}$ is the stopping power in silicium (from SRIM).

Figure 9 shows the calculated molecular release rates (Eq. (16)) as a function of the column density for the initial CR $E^{-1}$ and SP68 spectra. By comparing those molecular release rates with the rate parametrized by the stopping power law, one finds that the behaviour is very close to that obtained with $\alpha=2$. It is well known that thermal whole grain heating is mainly induced by very heavy CRs.

\section{Conclusions}

Both ionization and material (grain) processing rates induced by cosmic ray slowing down in a $\mathrm{H}_{2}$ molecular cloud were computed. The chemical composition of the CR was explicitely taken into account in the slowing down process and in the cross section determinations. We used two different types of CR spectra. One spectra contained few and the other many low-energy cosmic rays. The ionization rates were compared to the classical approximation (i.e. increment of the proton ionization rates by a constant factor representing the other CR species). For the spectrum containing many low-energy CRs, the net effect of the full calculation was a reduction of the classical ionization rates of 30 percent or less.

We studied the variation of the material processing rates between the outer and inner part of the cloud. For modification processes proportional to the stopping power, such as covalent bond destruction or ice compaction, the rates were found to be identical everywhere in the cloud in the case of a spectrum with weak low-energy CRs content, while with the spectrum with strong low-energy content, the rates are attenuated by one order of magnitude at column density of $10^{23} \mathrm{H}_{2} / \mathrm{cm}^{2}$, as were the ionization rates. For rates proportional to the square of the stopping powers, such as direct or thermal desorption, the attenuation at the same column density was found to be 2 in the case of spectrum with weak low-energy CR content, and two orders of magnitude for the other spectrum.

In this paper, we did not take the magnetization of the cloud into account, although this is fully relevant to the penetration of charge particles. For example, to mimic the attenuation by self-generated Alfvén waves, one calculates ionization rates with a low-energy cut (in Eq. (13)), corresponding to the magnetic 
M. Chabot: Cosmic-ray slowing down in molecular clouds: Effects of heavy nuclei

rigidity of $1 \mathrm{MeV}$ proton. Then, using a spectrum with a high content of low-energy CRs, the heavy CR are the dominant species at the entrance of cloud while they are a minor species deep inside the cloud. Indeed, as compared to protons, heavy ions are less sensitive to the magnetic field, but also experience a large stopping power. Studies explicitly coupling the slowing down of CR heavy species in a magnetized cloud are therefore needed.

Acknowledgements. Many thanks to Karine Béroff for her careful reading and improvement of the manuscript. Emmanuel Dartois and Vincent Tatischeff are thanked for the many discussions on that subject, still in progress. Thank to the referee for his work and to Jonathan Wilson for improving the English.

\section{References}

Betz, H.-D. 1972, Rev. Mod. Phys., 44, 465

Cecchi-Pestellini, C., \& Aiello, S. 1992, MNRAS, 258, 125

Chabot, M., Wohrer, K., Chetioui, A., et al. 1994, J. Phys. B, 27, 111

Chabot, M., Gardès, D., Nectoux, M., Maynard, G., \& Deutsch, C. 2007, Nucl. Instruments and Methods in Phys. Res. A, 577, 353

Cravens, T. E., \& Dalgarno, A. 1978, ApJ, 219, 750

Dartois, E., Ding, J. J., de Barros, A. L. F., et al. 2013, A\&A, 557, A97

Dartois, E., Augé, B., Boduch, P., et al. 2015, A\&A, 576, A125

D'Hendecourt, L. B., Allamandola, L. J., Baas, F., \& Greenberg, J. M. 1982, A\&A, 109, L12

George, J. S., Lave, K. A., Wiedenbeck, M. E., et al. 2009, ApJ, 698, 1666

Glassgold, A. E., \& Langer, W. D. 1973, ApJ, 186, 859

Godard, M., Féraud, G., Chabot, M., et al. 2011, A\&A, 529, A146

Indriolo, N., Geballe, T. R., Oka, T., \& McCall, B. J. 2007, ApJ, 671, 1736

Indriolo, N., Fields, B. D., \& McCall, B. J. 2009, ApJ, 694, 257
Kaganovich, I. D., Startsev, E., \& Davidson, R. C. 2006, New J. Phys., 8, 278 Leger, A., Jura, M., \& Omont, A. 1985, A\&A, 144, 147

Lequeux, J., Falgarone, E., \& Ryter, C. 2002, Le milieu interstellaire, Savoirs actuels (EDP Sciences)

McGuire, J. H., Stolterfoht, N., \& Simony, P. R. 1981, Phys. Rev. A, 24, 97

Molvik, A. W., Kollmus, H., Mahner, E., et al. 2007, Phys. Rev. Lett., 98, 064801

Muñoz, A., Oller, J. C., Blanco, F., Gorfinkiel, J. D., \& García, G. 2007, Chem. Phys. Lett., 433, 253

Opal, C. B., Peterson, W. K., \& Beaty, E. C. 1971, J. Chem. Phys., 55, 4100

Opal, C. B., Beaty, E. C., \& Peterson, W. K. 1972, Atomic Data, 4, 209

Padovani, M., Galli, D., \& Glassgold, A. E. 2009, A\&A, 501, 619

Rudd, M. E., Goffe, T. V., Dubois, R. D., Toburen, L. H., \& Ratcliffe, C. A. 1983, Phys. Rev. A, 28, 3244

Rudd, M. E., Kim, Y.-K., Madison, D. H., \& Gay, T. J. 1992, Rev. Mod. Phys., 64,441

Shen, C. J., Greenberg, J. M., Schutte, W. A., \& van Dishoeck, E. F. 2004, A\&A, 415,203

Spitzer, Jr., L., \& Tomasko, M. G. 1968, ApJ, 152, 971

Stier, P. M., \& Barnett, C. F. 1956, Phys. Rev., 103, 896

Stolterfoht, N., Dubois, R. D., \& Rivarola, R. D. 1997, Electron emission in heavy ion-atom collisions, Springer atoms plasmas (Berlin: Springer)

Stone, E. C., Cummings, A. C., McDonald, F. B., et al. 2013, Science, 341, 150

Straub, H. C., Renault, P., Lindsay, B. G., Smith, K. A., \& Stebbings, R. F. 1996, Phys. Rev. A, 54, 2146

Tatischeff, V., Decourchelle, A., \& Maurin, G. 2012, A\&A, 546, A88

Vladimirov, A. E., Digel, S. W., Jóhannesson, G., et al. 2011, Comput. Phys. Comm., 182, 1156

Webber, W. R., \& Yushak, S. M. 1983, ApJ, 275, 391

Williams, J. F. 1967, Phys. Rev., 153, 116

Wohrer, K., Chetioui, A., Rozet, J. P., et al. 1986, J. Phys. B, 19, 1997

Wohrer, K., Chabot, M., Touati, A., \& Watson, R. L. 1994, Nucl. Instrum. Methods Phys. Res. B, 88, 174

Ziegler, J. F., Ziegler, M. D., \& Biersack, J. P. 2010, Nucl. Instrum. Methods Phys. Res. B, 268, 1818 